\title{
ADVANCED MICROELECTRONICS TECHNOLOGIES FOR FUTURE SMALL SATELLITE SYSTEMS
}

\author{
Leon Alkalai \\ Center for Integrated Space Microsystems (CISM) \\ Jet Propulsion Laboratory \\ California Institute of Technology
}

\begin{abstract}
Future small satellite systems for both Earth observation as well as deep-space exploration are greatly enabled by the technological advances in deep sub-micron microelectronics technologies. Whereas these technological advances are being fueled by the commercial (non-space) industries, more recently there has been an exciting new synergism evolving between the two otherwise disjointed markets. In other words, both the commercial and space industries are enabled by advances in low-power, highly integrated, miniaturized (low-volume), lightweight, and reliable real-time embedded systems. Recent announcements by commercial semiconductor manufacturers to introduce Silicon On Insulator (SOI) technology into their commercial product lines is driven by the need for high-performance low-power integrated devices. Moreover, SOI has been the technology of choice for many space semiconductor manufacturers where radiation requirements are critical. This technology has inherent radiation latch-up immunity built into the process, which makes it very attractive to space applications. In this paper, we describe the advanced microelectronics and avionics technologies under development by NASA's Deep Space Systems Technology Program (also known as $\mathrm{X} 2000$ ). These technologies are of significant benefit to both the commercial satellite as well as the deep-space and Earth orbiting science missions. Such a synergistic technology roadmap may truly enable quick turn-around, low-cost, and highly capable small satellite systems for both Earth observation as well as deep-space missions.
\end{abstract}

\section{INTRODUCTION}

NASA's drive towards more frequent smaller missions (faster-better-cheaper) for both space science as well as Earth science is revolutionizing many aspects of space exploration. One benefit of having more frequent missions is the opportunity for more frequent technology insertion. That is, if a mission takes $5-10$ years to build and launch, the technology used may be commercially obsolete by the time of launch. On the other hand, having multiple launches a year enables new technology insertion as it becomes mature. This is especially true for the fast developing field of commercial microelectronics technologies.

The United States National Technology Roadmap for Semiconductors [1] still projects the continued reduction of the minimum semiconductor device features size $(\lambda)$ by a factor of $s=\sqrt{2} / 2$ every three years up to 2012. This market and technology- 
driven phenomenon, referred to as Moore's Law, was first observed by Gordon Moore, one of the co-founders of Intel Corporation, in the early 60s. Sustained reduction of the minimum feature size enables higher levels of functional integration on a single chip (integrated circuit). Moreover, higher volumes of production, better manufacturing capabilities and improved reliability leads to lower cost. Therefore, the net result: more for less, has been the main fuel behind the microelectronics technological revolution of the $20^{\text {th }}$ century.

Because of the uniquely harsh requirements of space applications (radiation, thermal, vibration, reliability, etc.), it was generally considered that commercial products based on commercial semiconductor technology are not readily applicable to space. Whereas this is generally correct, recent trends both in the commercial consumer sector, as well as in the space science and technology sector, are moving the two (otherwise disjoint) underlying technologies closer together. For example, portable and mobile computing platforms in the consumer industries are stimulating the development of low-power, energy-efficient, reliable, high-performance, embedded (real-time), highly miniaturized, and light-weight, modular and upgradable computer systems. All of these attributes have become essential requirements in the development of low-cost space science and commercial satellite systems.

In this paper, we initially describe a new program at NASA designed to develop new mission-enabling spacecraft systems every three years. This program called Deep Space Systems Technology Program, and commonly referred to as X2000, is managed at NASA's Jet Propulsion Laboratory. A major emphasis of X2000 is the development and insertion of new microelectronics and micro-avionics technologies into future missions. This work is performed by the newly formed Center for Integrated Space Microsystems (CISM) [2] described in Section 3. In Section 4, we describe the X2000/CISM microavionics technology roadmap through three generations of spacecraft systems. Finally, we offer our concluding remarks in Section 5.

\section{NASA'S DEEP SPACE SYSTEMS PROGRAM (X2000)}

Starting in fiscal year 1998, NASA initiated a new focused deep-space systems technology program as part of the NASA Office of Space Science Directorate. This program, also referred to as $\mathrm{X} 2000$, has as its goal to develop new generations of spacecraft systems, on three year intervals, that will enable future deep-space science missions [3]. Functionally, the program has three major elements: 1) an element focused on delivering new generations of flight-ready spacecraft systems called: X2000 1st, 2nd, 3rd , ... Delivery Projects; 2) a Center for delivering Integrated Space Micro-systems (CISM) for the flight-systems as well as research micro-avionics technologies for future missions; and 3) a program focused on delivering new Advances in Radio-isotopic Power Sources (ARPS) [4]. The X2000 Ist Delivery Project is well under way [5], whereas the 2 nd Delivery Project is expected to be initiated later this year. One of the goals of the X2000 Program as a whole, is to make a major technology push towards highly integrated Systems On A Chip. This technology is part of the newly formed center CISM, described in the following section. 


\section{CENTER FOR INTEGRATED SPACE MICROSYSTEMS (CISM)}

The newly formed center for integrated space microsystems (CISM) is an element of the X2000 Program, as well as a Center of Excellence at the Jet Propulsion Laboratory [6]. CISM currently supports the following three major technology thrust initiatives:

1. Near-Term avionics deliverables to flight projects.

2. Systems On A Chip (mid-term research) technologies for future flight projects.

3. Revolutionary Computing Technologies (long-term, basic research) with potential breakthroughs in 10 years or beyond.

\subsection{Near-Term Research and Development}

The avionics architecture, shown in Figure 1 below, for the 1st Delivery Project has already been selected, with the architectural baseline established at the Avionics Preliminary Design Review (PDR) held in August 1998. Even though revisions to the proposed baseline architecture are still being considered, the essential components are expected to remain the same. The X200 avionics shown in Figure 1 implements the main spacecraft control, communications, and monitoring functions. That is, it implements the traditional spacecraft functions of a Command Data Handling Subsystem (CDH), the Power Control and Distribution Subsystem, Attitude Control, Signal Processing, etc. This avionics architecture is both general enough and scaleable to accommodate flight projects of different requirements, including: Europa Orbiter, New Millennium Program Deep Space 4, Mars Sample Return, Pluto/Kuiper Express, Solar Probe, and others. The 1st Delivery Avionics architecture is characterized by the following attributes:

- A Distributed, symmetric architecture, with computing nodes and device nodes sharing a common bus architecture. The number of nodes and devices on the bus are scaleable, [7].

- Use of exclusively commercial off the shelf (COTS) interfaces and intellectual properties (IP) for their implementation on a radiation-hardened semiconductor foundry. The COTS interfaces include: 1394, IIC, JTAG, and PCI local bus. The buses are redundant in the baseline.

- Next generation System Flight Computer (SFC) with an order of magnitude improvement over existing state of the art computer performance. A baseline target performance of $>100$ MIPS (goal 200 MIPS) is under development. The advanced 32-bit computer will be built in a standard Compact PCI $3 \mathrm{U}$ format. This will facilitate and reduce the cost of early development, system integration and test, as well as the avionics system recurring cost.

- Next generation integrated packaging technology using Compact PCI (CPCI) backplane technology with enhancements for conductive cooling. This packaging is compliant with the standard CPCI backplane.

- Next generation micro-controller technology for embedded intelligent devices.

- Advanced integrated non-volatile memory systems.

- Highly integrated Power Activation and Switching Modules using solid state devices and High Density Interconnect technologies.

- Advanced Silicon On Insulator (SOI) semiconductor technology for low-power, high-performance integrated circuit design. 
- Advanced digital ASIC technology for over 1.0-1.5 M gates integrated on a single chip.

- Advanced mixed/signal ASIC technology for integrated power electronics.

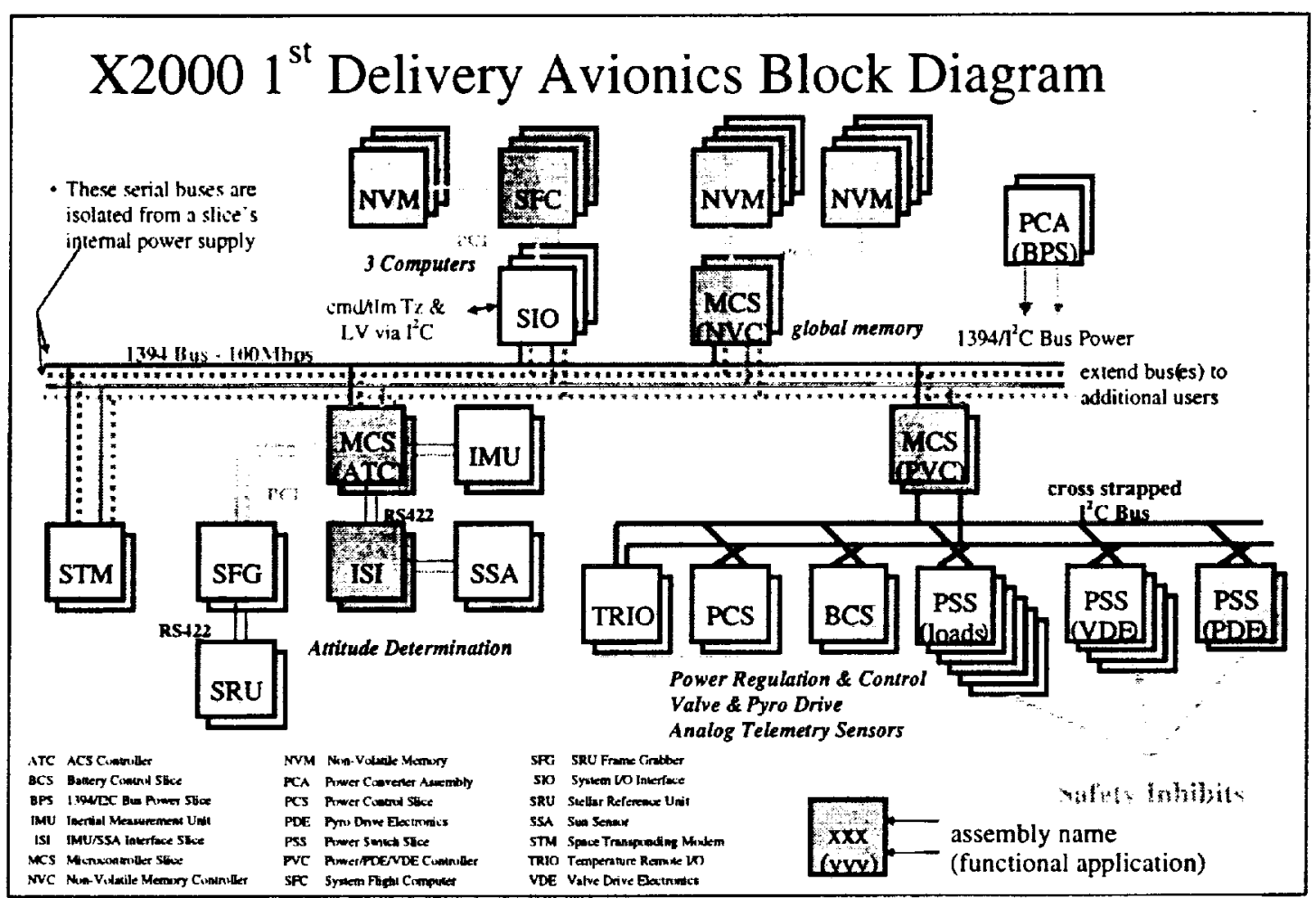

Figure 1. X2000 $1^{\text {st }}$ Delivery Avionics Architecture Block Diagram

\subsection{Systems On A Chip: Mid-Term R\&D}

The Systems On A Chip program targets future X2000 deliverables, starting with the $2^{\text {nd }}$ in 2003 [8], and the $3^{\text {rd }}$ in 2006. The elements of this research program include:

- RF Front-End miniaturization using micro-machining and thin film technologies.

- Micro Inertial Reference Systems (IRS) using MEMS technology.

- Integrated Multifunctional Imaging using Active Pixel Sensor (APS) technology.

- Integrated Passive Components using thin film micro-magnetics.

- Highly integrated power electronics using mixed signal ASIC technologies.

- Integrated sensor technologies for environmental, chemical, biological sensing and monitoring.

- Low Power devices, circuits, architectures, and components.

- Advanced design, integration and test technologies for systems on a chip.

- Design for high-yield and high-reliability; co-design and co-verification methodologies.

- And other integrated microsystems technologies.

- Design reuse for Systems On a Chip, using commercial IPs, soft cores, etc.

\subsection{Revolutionary Computing Technologies: Basic Research}


The Revolutionary Computing Technologies program is addressing basic research problems for future long-term computing needs in space applications. Several 'Grand Challenge' problems have been identified in this area:

- Sensing of Bio-Molecular Signatures for the identification of life.

- Ultra Reliable, Survivable microelectronics for long-term inter-stellar exploration, with survivability goals of $40-100$ years in outer space.

- Evolvable hardware circuits and microsystems.

- Quantum Functional Devices: Quantum Dots for low-power highly integrated systems.

- Quantum Computation and Communications.

- Optical Computing, Storage, and Communications.

- Biological Computing: DNA Computing.

- Bio-Mimetics, Bio-Informetics, etc.

In Table 1 below, we present an integrated technology roadmap for microelectronics sytems throughout the $3 \times 2000$ deliveries. Whereas the $1^{\text {st }}$ Deliverable is somewhat firm, the $2^{\text {nd }}$ and especially the $3^{\text {rd }}$ Deliverable are currently being studied. Therefore, the inputs for these are only tentative, and are most certainly going to change.

\section{SYNERGISM WITH CONSUMER ELECTRONICS AND COMMERCIAL SPACE SECTORS}

More than ever before, it seems that the world of consumer microelectronics, commercial space systems, as well as deep-space and Earth orbiting science missions, have a consistent technology roadmap. In other words, all of these markets are greatly enabled by the development of technologies for higher levels of integration, miniaturization, reduction in mass, volume and power, higher reliability and on-board intelligence (autonomy) $[9 ; 10]$. Moreover, from the semiconductor technology level, up to the avionics systems level, there seem to be very few points of departure.

Silicon On Insulator (SOI) technology has increasingly been the technology of choice for space applications due to its inherent immunity to single-event latch-up from high-energy particles. In fact, a low-power SOI experiment based on the 0.25 micron technology from MIT/LL is currently being validated on board the New Millennium DS1 mission [11]. The same technology has recently been announced as the choice for commercial semiconductor manufacturers due to its applicability to low-power highperformance devices.

Even at the component level, such as in the case of commercial DRAM technologies, newer generations of components such as $16 \mathrm{Mbit}$ and $64 \mathrm{Mbit}$ DRAMs, are no less prone to Single Event Upsets (SEU). This is because even at the commercial level, a floor has been reached due to the experience of SEUs caused by alpha particles on ground [12].

As demonstrated by the X2000 Program, more and more COTS technology is being used in space systems. The challenge for radiation intensive missions like Europa, is to use COTS IPs combined with radiation hard foundries, and minimum redesign of the interfaces, to achieve high levels of system reliability and radiation tolerance. Thus, at 
the architectural level, the system is compatible with commercial designs. This will reduce system development time and cost, as well as reduce the total system recurring cost.

\begin{tabular}{|c|c|c|c|}
\hline Technology & $1^{\text {st }}$ Delivery (2000) & $2^{\text {nd }}$ Delivery (2003) & $3^{\text {rd }}$ Delivery (2006) \\
\hline Semiconductor & & & \\
\hline feature size & $0.25 u m-0.35 u m$ & $0.18 u m-0.25 u m$ & $0.13 u m-0.18 u m$ \\
\hline process type & Bulk and SOI CMOS & SOI PD CMOS & SOI FD CMOS, SiGe \\
\hline supply voltage & $3.3-2.5$ volts & $2.5-1.5$ volts & $1.5-0.9$ volts \\
\hline LM (wafer size) & $4-5\left(6^{\prime \prime}-8^{\prime \prime}\right)$ & $5-6\left(8 "-10^{\prime \prime}\right)$ & $6-8\left(10^{\prime \prime}-12^{\prime \prime}\right)$ \\
\hline \multicolumn{4}{|l|}{ RH Building Blocks } \\
\hline CPU & PPC $750 / 740,603 \mathrm{e}$, Pentium & 32 bit CPU Core & 64 bit - CPU Core \\
\hline Micro-controller & 32-bit high-performance & 32-bit core & 64-bit micro-controller core \\
\hline SRAM & 4 Mbit & 16 Mbit & 64 Mbit \\
\hline ASIC: digital & $1.0-1.5 \mathrm{M}$ gates $(0.35 \mathrm{um})$ & $1.5-4.0 \mathrm{M}$ gates $(0.25 \mathrm{u})$ & $4.0-8.0 \mathrm{M}$ gates \\
\hline ASIC: analog/mixed & 0.8 um SOI mixed signal & 0.35 SOI mixed signal & 0.25 SOI mixed signal \\
\hline DRAM: commercial & 16,64 Mbit & $64-256 \mathrm{Mbit}$ & $256 \mathrm{Mb}, 1 \mathrm{Gbit}$ \\
\hline Non-Volatile: Flash, etc. & 64 Mbit & 256 Mbit & 1 Gbit \\
\hline \multicolumn{4}{|l|}{ COTS Interfaces } \\
\hline Local Bus & PCI $2.1 @ 33 \mathrm{Mhz}$, 64-b & High-Rel PCI & On-Chip PCI Core \\
\hline Low Power Utility Bus & $\mathrm{I} 2 \mathrm{C}$ & $\mathrm{I} 2 \mathrm{C}$ & Distributed I2C \\
\hline Eng. Bus & $1394100 \mathrm{Mbps}$ & $1394400 \mathrm{Mbps}$ & Multi-fiber optic links \\
\hline High-Speed Bus & IEEE 1394 & 1 Gbps X-bar Switches & On-chip X-bar Switches \\
\hline Test Bus & IEEE 1149.1 JTAG & IEEE $1149.1 / 5$ JTAG & Macro Cell \\
\hline \multicolumn{4}{|l|}{ Advanced Packaging } \\
\hline Avionics Systems Packaging & $\begin{array}{l}\text { Standard Compact } \\
\text { backplane (box) }\end{array}$ & $\begin{array}{l}\text { Horizontally Mounted } \\
\text { Cube (HMC) of Slices }\end{array}$ & $\begin{array}{l}\text { Microelectronics embedded } \\
\text { into multifunctional } s / c\end{array}$ \\
\hline Board Level packaging & $\begin{array}{l}3 \mathrm{U} \text { Compact PCI boards } \\
\text { with conductive cooling }\end{array}$ & $\begin{array}{l}5 " \times 5 \text { " slices with } \mathrm{Z} \text { and } \\
\mathrm{Y} \text { axis interconnects }\end{array}$ & $\begin{array}{l}\text { Distributed slices with flex } \\
\text { interconnects in structure }\end{array}$ \\
\hline Chip Scale Packaging & $\begin{array}{l}\text { BGA, C4, Chip on Board, } \\
\text { MCMs }\end{array}$ & $\begin{array}{l}\text { BGA, C4, Chip on } \\
\text { Board, MCMs }\end{array}$ & $\begin{array}{l}\text { BGA, C4, Chip on Board, } \\
\text { MCMs }\end{array}$ \\
\hline 3D Chip Stacking & $\begin{array}{l}4-48 \text { IC per } 3 D \text { memory } \\
\text { stack }\end{array}$ & $\begin{array}{l}\text { Memory and other IC in } \\
\text { stack }\end{array}$ & $\begin{array}{l}\text { Design for high-density 3D } \\
\text { stack integration of ICs }\end{array}$ \\
\hline \multicolumn{4}{|l|}{ Design Automation } \\
\hline CAD & $\begin{array}{l}\text { VHDL IC Models, Soft-Core } \\
\text { IPs transfer to RH foundries }\end{array}$ & $\begin{array}{l}\text { System-Level } \\
\text { Models, RH IPs }\end{array}$ & $\begin{array}{l}\text { System Level modeling and } \\
\text { synthesis }\end{array}$ \\
\hline Collaborative Eng. & Distributed Design & Virtual Skunk-works & Language to Layout \\
\hline \multicolumn{4}{|l|}{ Attitude Control } \\
\hline Star Tracker & Adv. Stellar Compass & Optical Processing & Integrated msystem \\
\hline $\begin{array}{l}\text { Sun Sensors/Gyros/Acc. } \\
\text { Fault Tolerance }\end{array}$ & MEMS-based & MEMS-based & MEMS-based \\
\hline FT Model & Distributed FT: $2-3$ nodes & Distributed FT (n-nodes) & Distributed FT \\
\hline SW FT Model & SW implemented FT & Design for Reliability & Design for Survivability \\
\hline Techniques & Adaptive FT & Self Repair & Evolving - Immune based \\
\hline Reliability Modeling & Partial Models & Integrated Tools Set & End-to-End Models \\
\hline \multicolumn{4}{|l|}{ Power Electronics } \\
\hline $\begin{array}{l}\text { Power activation and } \\
\text { switching modules (PASM) }\end{array}$ & 16 solid state power switches & 48 Switches & Switches on chip \\
\hline $\begin{array}{l}\text { Power management and } \\
\text { distribution (PMAD) }\end{array}$ & High Efficiency DC/DC & High Efficiency DC/DC & PASM/PMAD on a Chip \\
\hline Integration approach & $\begin{array}{l}\text { High Density Interconnect } \\
\text { (HDI) }\end{array}$ & $\begin{array}{l}\text { High Density } \\
\text { Interconnect (HDI) }\end{array}$ & $\begin{array}{l}\text { Macro-cells integrated on a } \\
\text { chip }\end{array}$ \\
\hline \multicolumn{4}{|l|}{ Telecom Processing } \\
\hline $\begin{array}{l}\text { I/F to Avionics } \\
\text { Enhancing Technologies }\end{array}$ & RF interface via 1394 bus & Optical Com. I/F & On Chip Interface \\
\hline Neural Networks & Autonomy support & S/C Navigation & Leaming Cells \\
\hline DSPs & SHARC21060 - HDSP & Ultra SHARC & Standard Cell \\
\hline
\end{tabular}




\section{CONCLUSIONS}

NASA's Deep Space Systems Technology Program, also known as X2000, is currently developing a series of advanced spacecraft systems for future deep-space science missions. An element of the X2000 Program is the newly formed Center for Integrated Space Microsystems (CISM). This center is responsible for the delivery of all spacecraft avionics to near-term missions launching in the 2003 time frame. Moreover, CISM is also placing major emphasis on future systems with technology development of Systems On A Chip, and Revolutionary Computing Technologies. The Avionics architecture described in this paper for the X2000 ${ }^{\text {st }}$ Delivery has numerous new technologies which are synergistic with the needs of the commercial satellite industries. Moreover, the technology roadmap for future space systems is compatible and synergistic in most ways, with recent developments in the commercial consumer electronics sectors. Examples include the use of advanced Silicon On Insulator (SOI) technology for integrated circuits, and components; commercial DRAM and Flash technology for volatile and non-volatile memory storage; Commercial Off The Shelf interfaces and IPs such as the 1394, IIC, 1149.1 JTAG, PCI, etc.; distributed, symmetric processing over a local-area network; and distributed fault-tolerance.

\section{REFERENCES}

1. The National Technology Roadmap for Semiconductors, SIA, 1997 Edition.

2. CISM web site: http://cism.jpl.nasa.gov

3. L. Deutsch, C. Salvo, and D. Woerner, NASA's X2000 Program - an Institutional Approach to Enabling Smaller Spacecraft, IAA $2^{\text {nd }}$ Annual Symposium on Small Satellites for Earth Observation (1998).

4. J. Mondt, Space Radiosiotope Power Source Requirements Update and Technology Status, Proceedings of the $33^{\text {rd }}$ Intersociety Energy Conversion Engineering Conference, (1998).

5. D. Woerner, X2000 Systems and Technologies for Missions to the Outer Planets, International Astronautical Federation Congress (1998)

6. L. Alkalai, A Roadmap for Space Microelectronics Technology into the New Millennium, Proceedings of the $35^{\text {th }}$ Space Congress (1998)

7. S. Chau, L. Alkalai, A. Tai, The Design of a Fault-Tolerant COTS-Based Bus Architecture for Space Applications, Submitted for publication, $29^{\text {th }}$ Annual International Symposium on Fault-Tolerant Computing (1999)

8. L. Alkalai, E. Kolawa, Avionics Systems On A Chip for Space Exploration, Proceedings of Space Technology \& Applications International Forum (STAIF-99). Opportunities and Challenges for the New Millenium (1999)

9. L. Alkalai, A. T. Tai, Long-Life Deep-Space Applications, Computer, IEEE, 31(4), 37-38 IEEE Computer Society, (1998).

10. A. Tai, L. Alkalai, S. Chau, "On-Board Preventive Maintenance: A Design-Oriented Analytic Study for Long Life Applications" To Appear, International Joumal Performance Evaluation, February 8, 1999.

11. L. Alkalai, "Advanced Flight Computing Technologies for Validation by NASA's New Millennium Program," Acta Astronautica, Journal of the International Academy of Astronautics, Vol. 39, No- 9. 12. pp 785-797, Nov. - Dec. 1996.

12. A. H. Johnston, "Radiation Effects in Advanced Microelectronics Technologies" IEEE Transactions on Nuclear Science, pp 1339-1354, June 1998. 


\section{ACKNOWLEDGEMENTS}

The work (or research) described in this paper was performed by the Jet Propulsion Laboratory, California Institute of Technology, under a contract with the National Aeronautics and Space Administration. This work is supported by NASA's Office of Space Science, Deep Space Systems Program, and JPL's Center for Integrated Space Microsystems. 